\title{
Spin susceptibility of neutron matter at zero temperature
}

\author{
S. Fantoni ${ }^{1,2}$, A. Sarsa ${ }^{1}$ and K. E. Schmidt ${ }^{3}$ \\ 1 International School for Advanced Studies, SISSA, I-34014 Trieste, Italy \\ 2 International Centre for Theoretical Physics, ICTP, I-34014 Trieste, Italy \\ ${ }^{3}$ Department of Physics and Astronomy, Arizona State University, Tempe, AZ 85287 U.S.A.
}

\begin{abstract}
The Auxiliary Field Diffusion Monte Carlo method is applied to compute the spin susceptibility and the compressibility of neutron matter at zero temperature. Results are given for realistic interactions which include both a two-body potential of the Argonne type and the Urbana IX three-body potential. Simulations have been carried out for about 60 neutrons. We find an overall reduction of the spin susceptibilty by about a factor of 3 with respect to the Pauli susceptibility for a wide range of densities. Results for the compressibility of neutron matter are also presented and compared with other available estimates obtained for semirealistic nucleon-nucleon interactions and with more traditional many-body techniques, like Brueckner's or Correlated Basis Function theories.
\end{abstract}

PACS numbers: 26.60.+c, 26.50.+x, 05.10.Ln 
In this paper we show that the strong correlations induced by realistic nucleon-nucleon interactions reduce, by up to about a factor of 3 , the spin susceptibility, $\chi$, of degenerate neutron matter. This reduction may have important implications for problems of astrophysical interest, like for instance, neutrino scattering rates in dense matter and, more generally, the study of supernovae and proto-neutron stars [1,2].

Starting from the pioneering work by Sawyer [3], several calculations of the neutrino mean free path in uniform nuclear matter have been performed [2: [4] which show that the effects due to strong interactions are relevant, particularly in the spin-density channel which couples with the axial vector current. A sizable reduction of $\chi$, or, equivalently, a large value of the $G_{0}$ Landau parameter leads to an appreciable suppression of the Gamow-Teller transitions.

The neutrino momenta and the momentum transfers in many applications are small compared with the neutron Fermi momentum, and both energy transfers and the temperature are small compared with the neutron Fermi Energy. Therefore, the Landau parameters of neutron matter at zero temperature are the main quantities to compute in order to evaluate the mean free path of a neutrino in dense matter.

In this respect, ab initio calculations of the Landau parameters or related quantities, such as the compressibility $\mathcal{K}$, the effective mass $m^{*}$ or the spin susceptibility $\chi$, for degenerate neutron matter are extremely important. Such calculations can now be performed because of recent advances in many-body methods, particularly those based on quantum simulations, and because of the much improved knowledge of the nucleon-nucleon interaction.

Previous evaluations of the Landau parameters $F_{l}$ and $G_{l}$ (with $l \leq 1$ ) for neutron matter were based either on Skyrme-type potential models [2] or on microscopic calculations performed with semirealistic bare interactions [7 9]. The qualitative behaviour of the compressibility ratio is

$$
\frac{\mathcal{K}}{\mathcal{K}_{F}}=\frac{1+\frac{1}{3} F_{1}}{1+F_{0}},
$$

where $\mathcal{K}_{F}$ the compressibility of the non interacting Fermi gas, is similar in most of the various calculations. On the contrary, the spin susceptibility ratio, which is approximately related to $G_{0}$ by

$$
\frac{\chi}{\chi_{F}} \approx \frac{1+\frac{1}{3} F_{1}}{1+G_{0}},
$$

may differ up to a factor of 3 at equilibrium density of nuclear matter $\rho_{0}=0.16 \mathrm{fm}^{-3}$, and even more at higher densities.

The Skyrme models generally predict much smaller values of $G_{0}$ with respect to microscopic calculations, and become unstable to spin oscillations, driving toward ferromagnetic ground states [2], since $1+G_{0}$ becomes negative for densities in the range $(2-4) \rho_{0}$. At small momentum transfer, $\mathrm{N}-\mathrm{N}$ correlations enhance the spin-response approximately by a factor $\left(\chi / \chi_{F}\right)^{2}$. Therefore, Skyrme models predict neutrino mean free paths smaller than microscopic models.

On the other hand, the existing microscopic calculations have been performed with old semirealistic interactions, like the Reid or the Bethe-Johnston potentials, and no threebody force. In addition, the many-body methods used until now may be questionable for 
the convergence of the underlying perturbation theory as well as for the treatment of spindependent correlations.

We report the results of quantum simulations of neutron matter for the old Reid- $v_{6}$ potential [10] and modern realistic interactions, based upon the Argonne $v_{18}$ two-body potential plus the Urbana three-body potential, UIX, [11], denoted hereafter as AU18. It is well known that such interactions provide a realistic description of light nuclei and nuclear matter [12, [13].

The quantum simulations have been carried out by using a new Diffusion Monte Carlo method, the so called AFDMC method [14]. It introduces auxiliary field variables in the simulation, which makes it the only existing quantum Monte Carlo method that can handle spin-dependent nuclear Hamiltonians and a relatively large number of nucleons. In this approach the scalar parts of the Hamiltonian are propagated as in standard Diffusion Monte Carlo (DMC) [15]. Auxiliary fields are introduced to replace the spin-isospin dependent interactions between pairs of particles with interactions between particles and auxiliary fields. Integrating over the auxiliary fields reproduces the original spin-isospin dependent interaction. The method consists of a Monte Carlo sampling of the auxiliary fields and then propagating the spin-isospin variables at the sampled values of the auxiliary fields. This propagation results in a rotation of each particle's spin-isospin spinor.

The guiding function, $\Psi_{T}$, in our AFDMC calculation is a simple trial function given by a Slater determinant of one-body space-spin orbitals multiplied by a central Jastrow correlation. The orbitals are plane waves that fit in the box times two component spinors, corresponding to neutron-up and neutron-down states. The overlap of a walker with this wave function is the determinant of the space-spin orbitals, evaluated at the walker position and spinor for each particle, and multiplied by the scalar Jastrow product. Such an overlap is complex, so the usual fermion sign problem becomes a phase problem. We constrain the path of the walkers to a region where the real part of the overlap with our trial function is positive. For spin-independent potentials this reduces to the fixed-node approximation.

This method has already been applied to unpolarized neutron matter and neutron drops ( $A=7,8)$ with fairly realistic interactions that include tensor, spin-orbit and three-body terms. The neutron matter calculations have been done with up to 66 neutrons in a periodic box with a low variance $(<0.1 \mathrm{MeV}$ per nucleon). The calculation scales in particle number roughly like fermion Monte Carlo with central forces [16,17].

We compute the spin susceptibility by applying a magnetic field to the system. Ignoring any orbital effects, the Hamiltonian is given by

$$
H=H_{0}-\sum_{i} \vec{\sigma}_{i} \cdot \vec{b}
$$

where $\vec{b}=\mu \vec{B}$ and $\mu=6.030774 \times 10^{-18} \mathrm{MeV} /$ Gauss, and the susceptibility is defined as

$$
\chi=-\left.\rho \mu^{2} \frac{\partial^{2} E_{0}(b)}{\partial b^{2}}\right|_{b=0},
$$

where $\rho$ is the number density and $E_{0}(b)$ is the ground energy in field $b$.

Let us use the Pauli expansion of the energy per particle as a function of the spin polarization $p=-\partial E_{0}(b) /\left.\partial b\right|_{b=0}$ 


$$
E(p)=E(0)-b p+\frac{1}{2} p^{2} E^{\prime \prime}(0)
$$

where the derivatives are with respect to the polarization. Minimizing $E(p)$ with respect to $p$ one gets the following result for the spin susceptibility

$$
\chi=\mu^{2} \rho \frac{1}{E^{\prime \prime}(0)} .
$$

For a noninteracting Fermi gas the spin susceptibility is $\chi_{F}=\mu^{2} m k_{f} /\left(\hbar^{2} \pi^{2}\right)$. AFDMC allows us to get the energy eigenvalue, $E_{0}\left(J_{z}, b\right)$, for the interacting system in a field $b$ for a state of a given spin asymmetry $J_{z}=N_{\uparrow}-N_{\downarrow}$.

Assuming that the energy and polarization are known in terms of $J_{z}, E^{\prime \prime}(0)$ in Eq. (6) can be obtained as a straightforward application of the chain rule

$$
E^{\prime \prime}(0)=\left[\frac{\partial p}{\partial J_{z}}\right]^{-2}\left\{\frac{\partial^{2} E_{0}}{\partial J_{z}^{2}}-\frac{\partial E_{0}}{\partial J_{z}}\left[\frac{\partial p}{\partial J_{z}}\right]^{-1} \frac{\partial^{2} p}{\partial J_{z}^{2}}\right\}
$$

Since we are calculating the lowest energy state, the derivative of the energy with respect to $J_{z}$ vanishes. Therefore this result reduces to

$$
E^{\prime \prime}(0)=\left[\frac{\partial p}{\partial J_{z}}\right]^{-2} \frac{\partial^{2} E_{0}}{\partial J_{z}^{2}}
$$

Let us consider the non interacting finite systems as a guide for the quantum simulations of the interacting ones. For such systems the energy is not a quadratic function of the external field $b$. In figure 1 1 we plot $E_{0}\left(J_{z}, b\right)$, as a function of $b$, for four different systems with a finite number $N \sim 60$ of noninteracting neutrons in a periodic box at $\rho=2 \rho_{0}$. The cases, for which we have done simulations, are shown in the figure, namely $\left(N_{\uparrow}, N_{\downarrow}\right)=(33,33)$, $(33,27),(57,7)$ and $(57,0)$. One can see that the various $E\left(J_{z}, b\right)$ are linear in $b$ and each of them is tangent to the Pauli parabola (which refers to infinite Fermi gas case) at some value $b_{0}$ of the field $b$. We also compare in the figure the Pauli parabola with the exact result for the Fermi gas at that density. They are very close up to $b \sim 50 \mathrm{MeV}$. Using Eq. (8), one gets $\chi / \chi_{F} \sim 1$ for $J_{z}=50$ and $J_{z}=57$.

In the interacting case, the derivatives in Eq. (\$) can be easily estimated by computing $E_{0}\left(J_{z}, b\right)$ with AFDMC, and using the following equations

$$
\begin{aligned}
\frac{\partial p}{\partial J_{z}} & \approx \frac{E_{0}\left(J_{z}=J_{z 0}, b=0\right)-E_{0}\left(J_{z}=J_{z 0}, b=b_{0}\right)}{J_{z 0} b_{0}}, \\
\frac{\partial^{2} E_{0}}{\partial J_{z}^{2}} & \approx 2 \frac{E_{0}\left(J_{z}=J_{z 0}, b=0\right)-E_{0}\left(J_{z}=0, b=0\right)}{J_{z 0}^{2}}
\end{aligned}
$$

whose validity relies on the following reasonable assumptions: (i) for $b=0, E_{0}\left(J_{z}, b\right)$ is quadratic in $J_{z}$; (ii) for a fixed $J_{z}, E_{0}\left(J_{z}, b\right)$ is linear in $b$; (iii) the polarization is linear in $J_{z}$. These assumptions become exact in the limit of an infinite system with $J_{z}$ and $b$ small.

The non-interacting case indicates the use of $J_{z 0}=50$ and the value for $b_{0}$ at which $E\left(J_{z 0}=50, b\right)$ is tangent to the Pauli parabola (for the density $\rho=2 \rho_{0}$ of Fig. 1, $b_{0}=53$ 
$\mathrm{MeV})$. Most of our calculations have been carried out with $J_{z 0}=50$. At $\rho=1.25 \rho_{0}$ we have verified the linearity of $E\left(J_{z 0}=50, b\right)$ on $b$ beyond $b_{0}\left(1.25 \rho_{0}\right)=39 \mathrm{MeV}$ finding that the result for $\chi / \chi_{F}$ is largely independent on the value of $b_{0}$. We have also verified the dependence of $\chi / \chi_{F}$ on $J_{z 0}$, performing simulations with $J_{z 0}=6$ and found that it is very weak.

A time step $\Delta \tau=5 \times 10^{-5} \mathrm{MeV}^{-1}$ was sufficient in most of the quantum simulations to obtain agreement between the mixed and the growth energies [15] within the statistical accuracy.

We have made simulations with the Reid $v_{6}$ interaction (Reid6), the same used in the Correlated Basis Function calculation of Ref. [9]. Our result for $\chi / \chi_{F}$, at $\rho=1.25 \rho_{0}$, is about $25 \%$ smaller, which indicates that the CBF perturbative calculations of Ref. [9], based on Jastrow-correlated basis functions, either have not reached a satisfactory convergence for the Landau parameter $G_{0}$ or Eq (2) may not be sufficiently adequate for the Reid6 interaction.

We have also considered realistic interactions, characterized by the so called $v_{6}^{\prime}$ or $v_{8}^{\prime}$ two-body potentials plus UIX three-body potential, and hereafter denoted as AU6' and AU8' respectively. The $v_{8}^{\prime}$ is a two-body potential of the Argonne type which includes the four central spin-isospin components, plus the four tensor and spin-orbit ones. It fits the nucleon-nucleon experimental data and embodies the main features of the Argonne $v_{18}$ [18]. The $v_{6}^{\prime}$ potential is the $v_{8}^{\prime}$ with its spin-orbit components removed.

Our results for $\chi / \chi_{F}$, obtained with the Reid6, AU6' and AU8' interactions are given in Table \and compared with previous microscopic calcuations of the same quantity. The Brueckner theory calculations of Ref. [8] are performed with the full Reid potential, and, therefore are not directly comparable with our Reid6 calculations. However, we do not find a sizable contribution to $\chi / \chi_{F}$ coming from the spin-orbit component of the two-body potential. Our results for AU6' and AU8' coincide within the statistical error. To address the problem of the influence of the three-body force on the results we have calculated the spin susceptibility at $\rho=2 \rho_{0}$ using the Argonne $v_{6}^{\prime}$ potential and no three body interactions. We have found that while the energy is reduced by roughly $25 \mathrm{MeV}$ per particle, the spin susceptibility ratio is practically unchanged $(0.31(1))$.

We have also calculated the compressibility $\mathcal{K}$, given by

$$
\frac{1}{\mathcal{K}}=\rho^{3} \frac{\partial^{2} E_{0}(\rho)}{\partial \rho^{2}}+2 \rho^{2} \frac{\partial E_{0}(\rho)}{\partial \rho},
$$

where $E_{0}(\rho)$ is a polynomial fit to the AFDMC energies $E\left(J_{z}=0, b=0\right)$. For a Fermi gas the compressibility is $\mathcal{K}_{F}=9 \pi^{2} m /\left(k_{f}^{5} \hbar^{2}\right)$. The AFDMC results for $\mathcal{K} / \mathcal{K}_{F}$, obtained with the AU6' interaction are shown in Table II, where they are also compared with the corresponding CBF estimates (AU6'-CBF) and other existing microscopic calculations [8,9, 13].

For the sake of completeness we show in Fig. 2 the equations of state of neutron matter, which have been used to compute the compressibility ratio of Table [1].

The AU18 results are taken from Ref. [13], and have been obtained with the full AU18 model interaction, by using variational FHNC/SOC methods. The AU6'-CBF [19] results have been obtained by using the AU6' interaction, as in the AFDMC simulations, and essentially the same many-body technique as in Ref. [13]. They also include the corrections coming from the lowest order elementary diagram, as discussed in Refs. [20, 17]. 
One can see that the AFDMC results for both the equation of state and the compressibility of the AU6' model of neutron matter are in reasonably good agreement with those obtained by using CBF theory.

We have estimated the finite size effects in the AFDMC simulations by performing variational calculations with the Periodic Box FHNC method of Ref. [21]. They indicate a correction which is at most $10 \%$ of the mixed energy per particle. An error of the same size is expected for the compressibilty ratio and of an order of magnitude smaller for the spin susceptibility. A more detailed discussion of the AFDMC calculation and the equation of state of neutron matter will be given elsewhere [17].

In conclusion, we have presented new results on the spin susceptibility and the compressibility of neutron matter at zero temperature, which show a strong reduction of these quantities with respect to their Fermi gas values. The calculations have been performed for realistic model interactions, which include tensor, spin-orbit and three-body terms, and by using the AFDMC method, a newly developed quantum simulation technique, capable of dealing with strongly spin-dependent interactions. Of particular relevance is the quenching by about a factor of 3 of the spin susceptibility with respect to the commonly used Pauli value, found at all the various densities considered. Such a reduction has strong effects on the mean free path of a neutrino in dense matter and should be seriously taken into account in the studies of supernovae and proto-neutron stars.

\section{ACKNOWLEDGEMENTS}

We are indebted to A. Fabrocini for providing us the results of the second order perturbative corrections to the energy per particle of neutron matter for the AU6' interaction

and to C.J. Pethick for insightful discussions. Portions of this work were supported by MURST-National Research Projects and CINECA computing center. 


\section{REFERENCES}

[1] G. G. Raffelt, Stars as Laboratories for Fundamental Physics, The University of Chicago Press, Chicago \& London (1996).

[2] S. Reddy, M. Prakash, J. M. Lattimer and J. A. Pons, Phys. Rev. C 59, 2888 (1999) and references therein.

[3] R. F. Sawyer, Phys. Rev. D 11, 2740 (1975); Phys. Rev. C 40,865 (1989).

[4] N. Iwamoto and C. J. Pethick, Phys. Rev. D 25, 313 (1982).

[5] B. L. Friman and O. V. Maxwell, Astr. Journal, 232, 541 (1979).

[6] A. Burrows and R. F.Sawyer, Phys. Rev. C 59, 510 (1999).

[7] J. W. Clark, Phys. Rev. Lett. 23, 1463 (1969).

[8] S. O. Bäckmann and C. G. Källman, Phys. Lett. B 43, 263 (1973).

[9] A. D. Jackson, E. Krotscheck, D. E. Meltzer and R. A. Smith, Nucl. Phys. A 386,125 (1992).

[10] R. V. Reid, Ann. of Phys. 50, 411 (1968).

[11] S. C. Pieper, in Microscopic Quantum Many-Body theories and their applications. Lecture Notes in Physics 510, (Springer Verlag, Berlin, 1998), p. 337.

[12] R. B. Wiringa, S. C. Pieper, J. Carlson and V. R. Pandharipande, Phys. Rev. C 62 014001 (2000).

[13] A. Akmal,V. R. Pandharipande and D. G. Ravenhall Phys. Rev. C 58, 1804 (1998).

[14] K. E. Schmidt and S. Fantoni, Phys. Lett. B 446, 99 (1999).

[15] K. E. Schmidt and M. H. Kalos, in Monte Carlo Methods in Statistical Physics II, Topics in Current Physics, ed. K. Binder (Springer-Verlag, Berlin, Heidelberg, New York, 1984) p. 125.

[16] S. Fantoni, A. Sarsa and K. E. Schmidt, Prog. Part. Nucl. Phys. 44, 63 (2000).

[17] K. E. Schmidt, J. Carlson, A. Sarsa, F. Pederiva and S. Fantoni, in preparation.

[18] A. Smerzi, D. G. Ravenhall and V. R. Pandharipande, Phys. Rev. C 56, 2549 (1997).

[19] A. Fabrocini, private communication.

[20] S. Fantoni, A. Sarsa and K.E. Schmidt, in Advances in Quantum Many-Body Theories, R.F. Bishop, K.A. Gernoth and N.R. Walet editors, (World Scientific, Singapore), in press.

[21] S. Fantoni and K. E. Schmidt, Nucl. Phys. A 690, 456 (2001). 


\section{FIGURES}

FIG. 1. The energy of non interacting neutrons as a function of magnetic field $b$ at $\rho=2 \rho_{0}$ for various finite-sized closed shell trial functions with spin up and down values shown. Also plotted is the correct infinite system energy and the parabolic Pauli value.

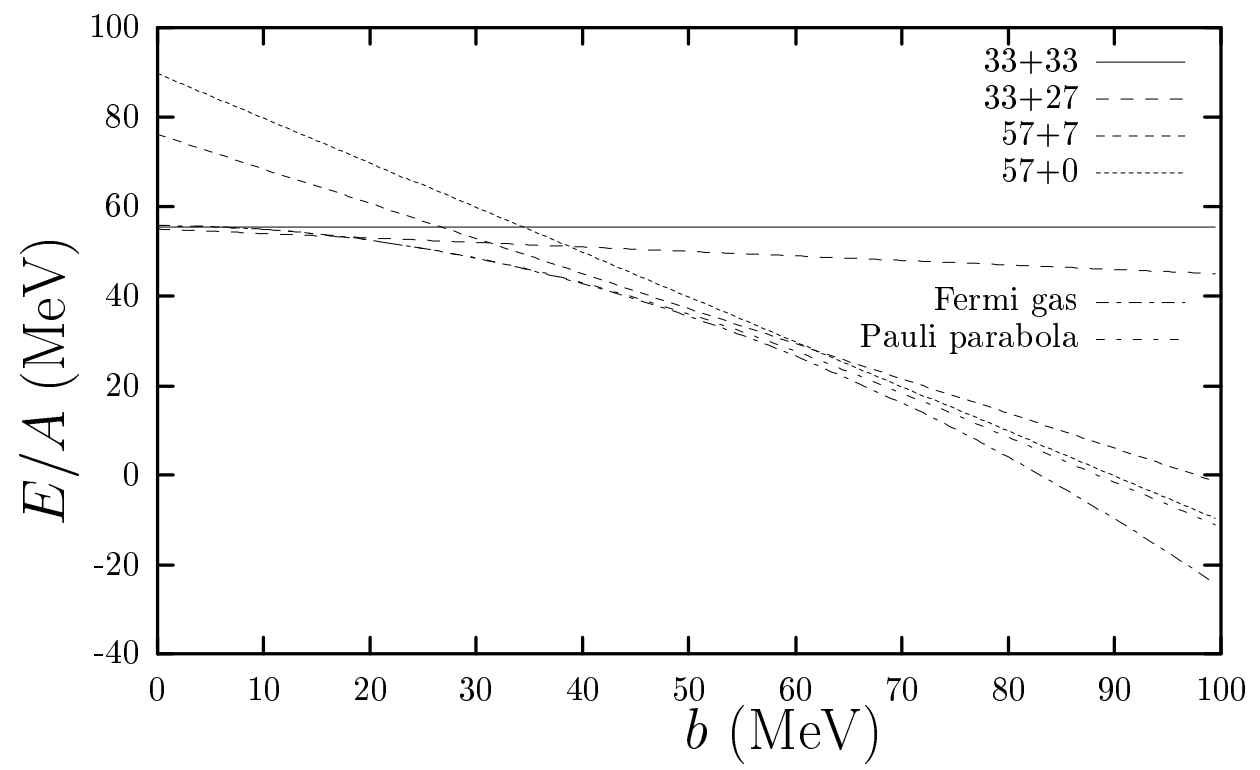

FIG. 2. AFDMC equation of state of the AU6' model of neutron matter (dots); CBF theory [19] results for the same interaction model are in the shaded area where the highest values correspond to the variational estimate. The equation of state obtained in Ref.[13] for the AU18 interaction by using FHNC/SOC theory is given by dashes. The errors are smaller than the symbols.

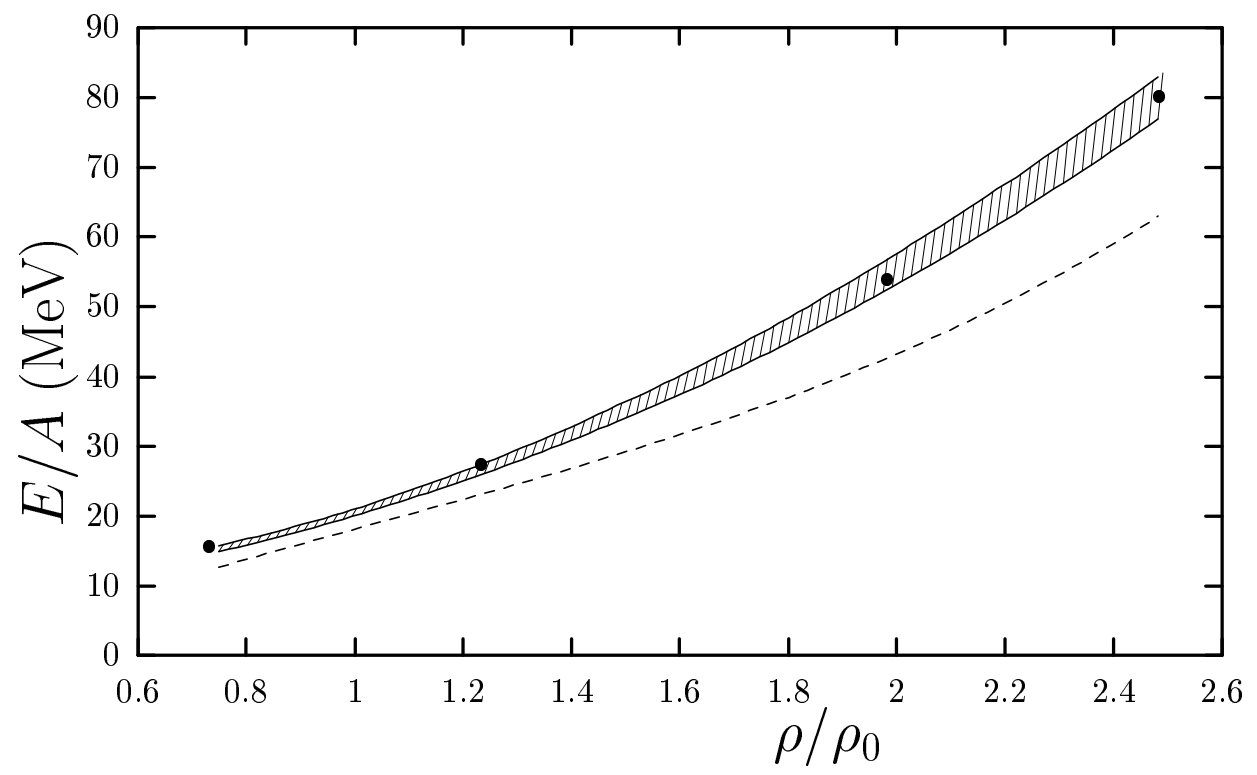




\section{TABLES}

TABLE I. Spin susceptibility ratio $\chi / \chi_{F}$ of neutron matter. Our AFDMC results for the interactions AU6', AU8' and Reid6 are compared with those obtained from Refs. [8,9] by using $\mathrm{Eq}(2)$. The statistical error is given in parentheses.

\begin{tabular}{c|cc|ccc}
\hline \hline$\rho / \rho_{0}$ & Reid [8] & Reid6 [9] & AU6' & AU8' & Reid6 \\
\hline 0.75 & 0.45 & 0.53 & $0.40(1)$ & & \\
1.25 & 0.42 & 0.50 & $0.37(1)$ & $0.39(1)$ & $0.36(1)$ \\
2.0 & 0.39 & 0.47 & $0.33(1)$ & $0.35(1)$ & \\
2.5 & 0.38 & 0.44 & $0.30(1)$ & & \\
\hline \hline
\end{tabular}

TABLE II. Compressibility ratio $\mathcal{K} / \mathcal{K}_{F}$ of neutron matter. Our AFDMC results for the AU6' interaction are compared with those obtained with CBF theory [19] (AU6'-CBF) and those of Refs. $[8,9,13]$ The statistical error is given in parentheses.

\begin{tabular}{c|ccc|cc}
\hline \hline$\rho / \rho_{0}$ & Reid [B] & Reid6 [9] & AU18 [13] & AU6'-CBF & AU6' \\
\hline 0.75 & 0.91 & 2.06 & 1.10 & 0.85 & $0.89(3)$ \\
1.25 & 0.70 & 1.35 & 0.71 & 0.45 & $0.47(3)$ \\
2.0 & 0.49 & 0.77 & 0.26 & 0.23 & $0.21(3)$ \\
2.5 & 0.42 & 0.60 & 0.15 & 0.17 & $0.14(3)$ \\
\hline \hline
\end{tabular}

